\title{
Atuação do fisioterapeuta na reabilitação de pacientes com síndrome da dor femoropatelar: Atualização das evidências científicas
}

\section{Physiotherapist activity in the rehabilitation of patients with femoropathic pain syndrome:}

\section{Update of scientific evidence}

Dayse Raquel Sousa da Silva ${ }^{1}$, Sarah Tiane Joodsie Costa da Silva²

\section{RESUMO}

A síndrome da dor femoropatelar é uma patologia que tem como principal característica a dor anterior do joelho, sua etiologia não é definida, podendo ser multifatorial com surgimento insidioso e se agravando em movimentos que provocam sobrecarga na articulação. A dor e as limitações durante a realização de atividades cotidianas são as principais queixas dos pacientes. O objetivo desse estudo foi expor as evidências científicas atuais da reabilitação fisioterapêutica do paciente com síndrome da dor femoropatelar. A revisão foi composta por artigos originais publicados entre 2013 e 2018 das bases de dados MEDLINE, PubMed, SciELO e LILACS com os seguintes descritores: síndrome da dor femoropatelar, fisioterapia, joelho e técnica de exercício e de movimento. Nove estudos preencheram os critérios com intervenções propostas para 0 tratamento fisioterapêutico. A técnica de cinesioterapia com enfoque em fortalecimento do quadril e joelho demonstrou resultados mais consistentes para diminuição da dor e melhora da capacidade funcional. As técnicas de Kinesio-Taping ${ }^{\circledR}$, órtese patelofemoral e estimulação nervosa elétrica apresentaram resultados positivos, porém em menor proporção sugerindo que sejam complementares à cinesioterapia.

Palavras-chave: Síndrome da dor femoropatelar. Fisioterapia. Joelho. Técnica de exercício e de movimento.

\section{ABSTRACT}

Patellofemoral pain syndrome is a pathology that has as its main characteristic anterior knee pain, its etiology is not defined, and may be multifactorial with insidious appearance and worsening in movements that cause overload in the joint. Pain and limitations during daily activity are the main complaints of patients. The objective of this study was to present the current scientific evidence of the physiotherapeutic rehabilitation of the patient with patellofemoral pain syndrome. The review was composed of original articles published between 2013 and 2018 from the MEDLINE, PubMed, SciELO and LILACS databases with the following descriptors: patellofemoral pain syndrome, physiotherapy, knee and exercise and movement technique. Nine studies fulfilled the criteria with interventions proposed for the physiotherapeutic treatment. The kinesiotherapy technique with focus on hip and knee strengthening demonstrated more consistent results for pain reduction and improvement of functional capacity. The Kinesio-taping techniques, patellofemoral orthosis and neuromuscular electrical stimulation presented positive results, but to a lesser extent suggesting that they are complementary to kinesiotherapy.

Keywords: Patellofemoral pain syndrome. Physiotherapy. Knee. Exercise technique and movement.
Fisioterapeuta, habilitada no Método TheraSuit.

E-mail:

dayseraquel17@gmail.com

2 Fisioterapeuta, Especialista em Gerontologia, Docente no Curso de Fisioterapia do Instituto de Ensino e Pesquisa Objetivo-IEPO 


\section{INTRODUÇÃO}

O joelho é uma das articulações mais estudadas e reabilitadas pela fisioterapia devido à relevância dessa articulação e ao número de lesões que ocorre nela. (SIZÍNIO, 2009; PEREIRA JÚNIOR, 2011; ZANARDI, 2012). Ao longo do tempo a fisioterapia desenvolveu formas e métodos para melhor tratar o joelho buscando aprofundar 0 conhecimento biomecânico e fisiológico (SIZÍNIO, 2009).

É a articulação de maior sobrecarga do corpo humano projetada para a mobilidade e estabilidade. Juntamente com quadril e pés suporta o corpo na posição ortostática e é uma unidade funcional primária nas atividades de andar, subir e descer escadas e sentar. É formado pela região distal do fêmur, região proximal da tíbia e patela (CAMPOS, 2013; BESSA et al., 2016).

A patela realiza duas importantes funções biomecânicas na articulação do joelho. Auxilia na extensão fazendo com que o tendão do quadríceps se desloque anteriormente, aumentando o braço de alavanca de força do quadríceps. Ela também atua distribuindo as forças de estresse que atuam no fêmur, ampliando, assim, a área de contato entre o tendão patelar e o fêmur permitindo uma melhora na distribuição da força de compressão (PEREIRA JÚNIOR, 2011; ASSIS, 2015).

Por ser uma articulação com grande sobrecarga, está mais suscetível a sofrer alterações e, consequentemente, o desenvolvimento de lesões. A síndrome da dor femoropatelar é uma patologia comum na população (PEREIRA JÚNIOR, 2011; ASSIS, 2015; PIAZZA, 2012) levando à diminuição de atividades físicas e ao aumento de faltas no trabalho, sendo caracterizada por dor na região anterior, peri e retropatelar, geralmente de início insidioso, piorando com atividades que exigem um aumento de forças de compressão na articulação patelofemoral (ASSIS, 2015). Não apresenta relação com acidente traumático e pode se manifestar acompanhada de crepitação, caracterizada pelo paciente como estalo e rangido durante os movimentos de flexão e extensão (SIZíNIO, 2009; SANTOS, 2013; SANCHEZ, 2017), rigidez, edema, fraqueza dos músculos extensores de joelho e responder positivamente ao sinal de Clarke e sinal de Rabot (ASSIS, 2015; SANTOS, 2013; ROQUE, 2012).

As causas da SDFP são consideradas multifatoriais e ainda não são definidas. Há correlacão de sua origem com alterações biomecânicas e estruturais dos membros inferiores, como a anteversão do colo do fêmur, o aumento da adução e rotação medial 
do quadril e desequilíbrios musculares do quadril e do joelho (OLIVEIRA, 2014). Fatores determinados biologicamente, como o aumento do ângulo $Q$, a hiperelasticidade manifestada em algumas pessoas, além de genum recurvatum, joelho valgo e alterações anatômicas como a patela mais alta, e a diminuição da força da musculatura do joelho deixam o indivíduo mais propenso para apresentar a SDFP. (CAMPOS, 2013; SANCHEZ, 2017).

É evidenciada durante atividades diárias como agachamento, permanência em posição sentada ou durante subida e descida de escadas. O principal sintoma é a dor e a condição geralmente evolui para comprometimento da função (ØSTERÅS et al., 2013) limitando a participação no esporte, caso o paciente seja atleta, ou provocando limitações nas atividades funcionais prejudicando a qualidade na locomoção dos pacientes acometidos, levando à diminuição de atividades físicas e ao aumento de faltas no trabalho.

Uma vez diagnosticada a SDFP, intervenções direcionadas aos elementos específicos do problema do paciente como algia e desequilíbrio muscular podem ser iniciadas (SIZÍNIO, 2009). O tratamento de primeira linha nos casos de SDFP é conservador que inclui um programa de reabilitação global e personalizado (ROQUE, 2012). O enfraquecimento da musculatura do joelho é um fator para a instalação da síndrome e diversas formas de intervenções são propostas para a resolução dessa disfunção. A fisioterapia dispõe de vários recursos bastante utilizados nessa doença como a cinesioterapia baseada em exercícios de fortalecimento e alongamento, métodos de terapia manual, bandagens e órteses (NETO, 2014; CAVALCANTE, 2014; PEREIRA JÚNIOR, 2014).

Como a patologia ocasiona limitações nas atividades funcionais, prejudicando a qualidade na locomoção dos pacientes acometidos pela mesma. A finalidade desse estudo é revisar na literatura as técnicas fisioterapêuticas utilizadas no tratamento, expondo as evidências científicas atuais da reabilitação fisioterapêutica de pacientes com síndrome da dor femoropatelar.

\section{MATERIAIS E METODOS}

Este estudo caracteriza-se por ser uma revisão de literatura com o objetivo de expor as evidências científicas atuais da reabilitação fisioterapêutica do paciente com SDFP, 
onde foram analisados artigos publicados nos últimos 5 anos. A busca foi realizada nas bases de dados LILACS, MEDLINE, PubMed e SciELO. Os descritores utilizados durante o processo de coleta de dados foram: "joelho", "síndrome da dor femoropatelar", "fisioterapia" e "técnica de exercício e de movimento". Os termos foram empregados de forma singular e combinados. Para análise, foram aplicados como critérios de inclusão artigos originais voltados ao tratamento conservador da SDFP, publicados no período de janeiro de 2013 a setembro de 2018 nos idiomas inglês, português e espanhol. Foram excluídos estudos que relacionam à SDFP a outra patologia, estudos com gestantes, artigos com pacientes amputados de membros inferiores, pesquisas com pacientes com histórico de lesão ou procedimentos cirúrgicos no quadril e joelho, pesquisas com pacientes em uso de medicação e fisioterapia prévia ao estudo. Realizou-se também a análise das referências bibliográficas dos artigos selecionados com o propósito de incrementar os dados da presente pesquisa.

A busca identificou noventa e quatro pesquisas nos bancos de dados e quatro em referências bibliográficas dos artigos selecionados. A ordem de seleção dos artigos está ilustrada no fluxograma a seguir. (Figura 1)

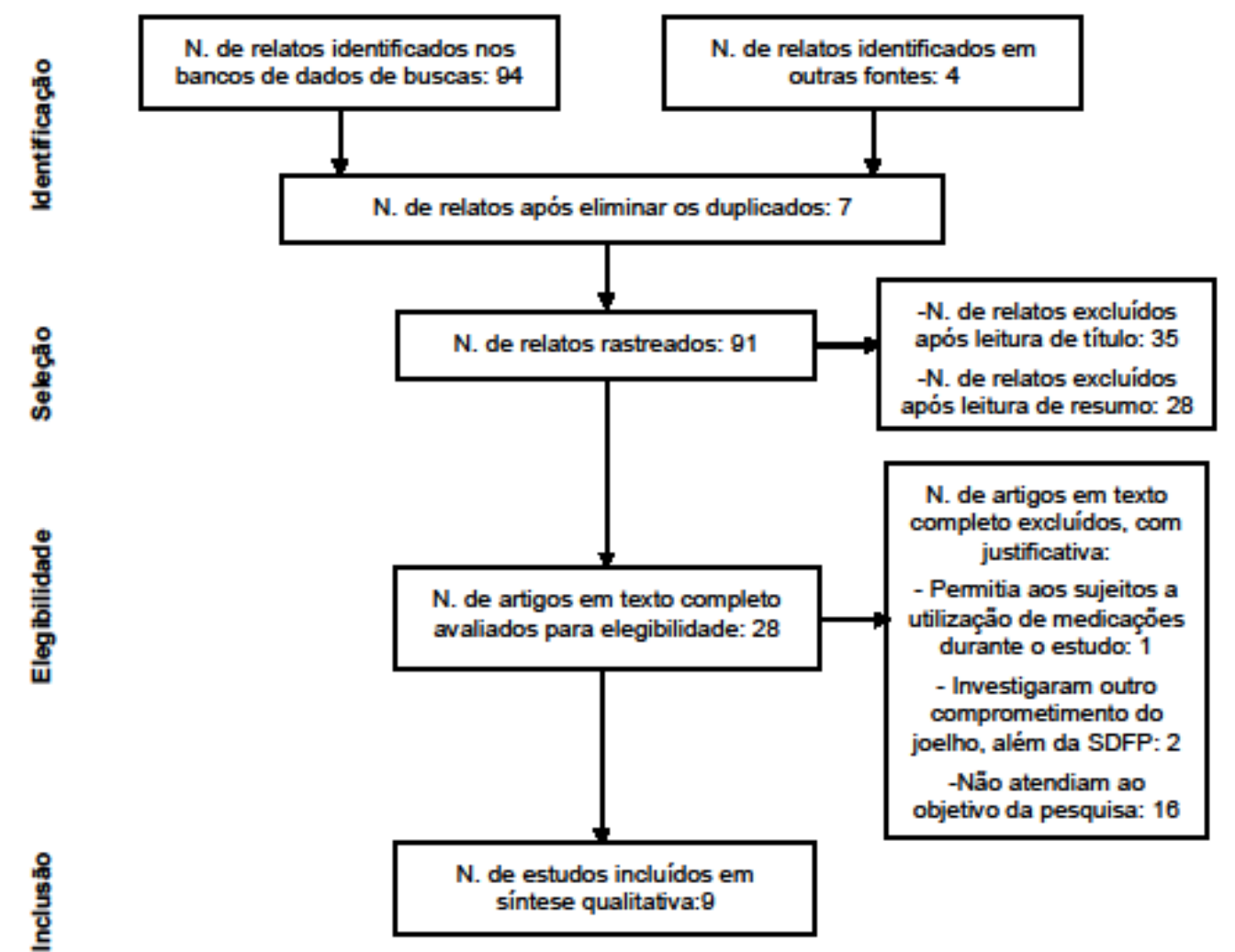

Figura 1. Seleção dos artigos 


\section{RESULTADOS}

Nove artigos foram selecionados para responder ao objetivo desta pesquisa, um na língua portuguesa e oito na língua inglesa. Os resultados estão descritos abaixo. (Tabela 1)

Tabela 1. Descrição dos estudos selecionados para análise final.

\begin{tabular}{|c|c|c|c|c|c|}
\hline Autor/Ano & $\begin{array}{l}\text { Tipo de } \\
\text { pesquisa }\end{array}$ & Sujeitos & Objetivo & $\begin{array}{l}\text { Abordagem } \\
\text { terapêutica }\end{array}$ & Conclusão \\
\hline $\begin{array}{l}\text { (ARAZPOU } \\
R \text { et al., } \\
2013 \text { ) }\end{array}$ & $\begin{array}{l}\text { Quase } \\
\text { experimental }\end{array}$ & $\begin{array}{l}6 \text { homens, } 4 \\
\text { mulheres }\end{array}$ & $\begin{array}{l}\text { Avaliar o efeito } \\
\text { da intervenção } \\
\text { na forma de } \\
\text { uma órtese de } \\
\text { controle da } \\
\text { patela na flexão } \\
\text { do joelho e } \\
\text { parâmetros } \\
\text { temporais } \\
\text { espaciais ao } \\
\text { caminhar }\end{array}$ & $\begin{array}{l}\text { Utilização de } \\
\text { órtese durante } \\
\text { pelo menos } 8 \\
\text { horas por dia } \\
\text { durante } \\
\text { semanas }\end{array}$ & $\begin{array}{l}\text { A utilização da órtese } \\
\text { de joelho resultou em } \\
\text { diminuição da dor, } \\
\text { melhora } \\
\text { parâmetros espaciais } \\
\text { temporais e aumento } \\
\text { dos ângulos de } \\
\text { flexão do joelho } \\
\text { durante } \\
\text { deambulação }\end{array}$ \\
\hline $\begin{array}{l}\text { (BALDON, } \\
\text { Rodrigo et } \\
\text { al., 2014) }\end{array}$ & $\begin{array}{l}\text { Ensaio } \\
\text { clínico } \\
\text { randomizado }\end{array}$ & $\begin{array}{l}31 \text { atletas } \\
\text { recreativas } \\
\text { do sexo } \\
\text { feminino. TP } \\
(n=16) \text { TEF } \\
(n=15)\end{array}$ & $\begin{array}{ll}\begin{array}{l}\text { Comparar } \\
\text { efeitos }\end{array} & \text { os } \\
\text { treinamento } & \\
\text { TEF x TP } & \end{array}$ & $\begin{array}{l}\text { TEF: aumentar } \\
\text { o controle } \\
\text { motor e força } \\
\text { dos músculos } \\
\text { do tronco e do } \\
\text { quadril. TP: } \\
\text { tratamento } \\
\text { focado no } \\
\text { fortalecimento } \\
\text { do quadríceps }\end{array}$ & $\begin{array}{l}\text { A intervenção TEF foi } \\
\text { mais benéfica na } \\
\text { melhora da dor, } \\
\text { função física, } \\
\text { cinemática e força } \\
\text { muscular em } \\
\text { comparação com a } \\
\text { intervenção TP }\end{array}$ \\
\hline $\begin{array}{l}\text { (FERBER } \\
\text { et } \\
\text { 2015) }\end{array}$ & $\begin{array}{l}\text { Ensaio } \\
\text { clínico } \\
\text { controlado } \\
\text { randomizado }\end{array}$ & $\begin{array}{l}66 \text { homens e } \\
133 \\
\text { mulheres }\end{array}$ & $\begin{array}{l}\text { Comparar os } \\
\text { protocolos } \\
\text { joelho e quadril } \\
\text { após } 6 \text { semanas } \\
\text { de reabilitação }\end{array}$ & $\begin{array}{l}\text { Os pacientes } \\
\text { aleatoriamente } \\
\text { designados } \\
\text { para um } \\
\text { protocolo } \\
\text { joelho } \\
\text { quadril de } 6 \\
\text { semanas }\end{array}$ & $\begin{array}{l}\text { O protocolo do } \\
\text { quadril resultou na } \\
\text { resolução do mais } \\
\text { precoce da dor e em } \\
\text { maiores ganhos } \\
\text { gerais de força em } \\
\text { comparação com o } \\
\text { protocolo do joelho }\end{array}$ \\
\hline $\begin{array}{l}\text { (GLAVIAN } \\
\text { O Neal; } \\
\text { SALIBA, } \\
\text { Susan } \\
\text { 2016) }\end{array}$ & $\begin{array}{l}\text { Investigação } \\
\text { laboratorial } \\
\text { randomizada } \\
\text { e duplo-cega }\end{array}$ & $\begin{array}{l}15 \text { mulheres } \\
\text { e } 7 \text { homens }\end{array}$ & $\begin{array}{l}\text { Determinar se } \\
\text { um tratamento } \\
\text { PENS alteraria } \\
\text { a atividade } \\
\text { muscular e a } \\
\text { dor durante um } \\
\text { agachamento } \\
\text { unipodal e um }\end{array}$ & $\begin{array}{l}\text { GE: } \\
\text { tratamento com } \\
\text { PENS durante } \\
15 \text { min. GC: } \\
\text { tratamento } \\
\text { subsensorial de } \\
1 \text { minuto }\end{array}$ & $\begin{array}{l}\text { Os participantes do } \\
\text { GE tiveram melhora } \\
\text { imediata na ativação } \\
\text { do glúteo médio e } \\
\text { redução da dor } \\
\text { durante as tarefas } \\
\text { funcionais }\end{array}$ \\
\hline
\end{tabular}




\section{(ØSTERÅS Estudo}

$\begin{array}{ll}\text { Berit et al., } & \text { controlado } 28 \text { indivíduos } \\ \text { 2013) } & \text { randomizado }\end{array}$

\section{Estudo}

(SAAD et randomizado simples-cego
40 mulheres al., 2018) controlado

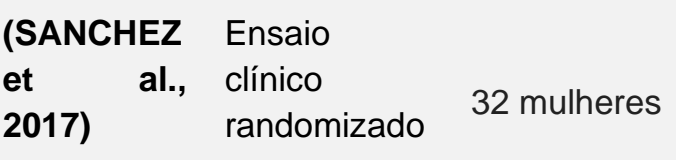

(SOUZA et Estudo al., 2017) prospectivo
24 mulheres. GSDFP: 14 . GC: 10 degrau lateral.

Avaliar o efeito a longo prazo da terapia com exercícios de alta dose e alta repetição

Avaliar o efeito e as contribuições de 3 tipos de intervenções para o controle da dor, função cinemática

\section{Avaliar} influência técnica Kinesio-Taping ${ }^{\circledR}$ na sintomatologia e funcionalidade

\section{Verificar a}

eficácia de um protocolo de fortalecimento de MMII em CCF.

$\begin{array}{lll}\text { (YELVAR } & \text { Estudo } \\ \text { et } & \text { al., } & \text { clínico } \\ \text { 2015) } & & \text { controlado } 42 \text { indivíduos } \\ & & \text { randomizado }\end{array}$

Legendas: TP: treinamento padrão; TEF: treinamento de estabilização funcional; GE: grupo experimental; GC: grupo controle; GQ: grupo quadril; GQS: grupo quadríceps; GA: grupo alongamento; GSDFP: grupo síndrome da dor femoropatelar; MMII: membros inferiores; CCF: cadeia cinética fechada; PENS: estimulação nervosa elétrica percutânea

GE: exercícios de alta dose e O GE continuou a alta repetição. melhorar enquanto o GC: exercícios GC recaiu com de baixa dose aumento de dor e e baixa disfunção repetição

GQ: exercícios de quadril. GQS:

exercícios de quadríceps.

GA:

alongamentos.

GC: sem intervenção

\section{Exercícios de} fortalecimento do quadril não foram mais eficazes redução da dor e melhora da função em comparação com exercícios de quadríceps ou alongamento

$\mathrm{O}$ uso de $\mathrm{KT}$ associado ao

$\begin{array}{lll}\text { Kinesio- } & \text { tratamento } \\ \text { Taping }{ }^{\circledR} \quad \text { e } & \text { fisioterapêutico }\end{array}$ fisioterapia convencional leva a convencional $x$ uma melhora na dor fisioterapia em comparação com convencional 0 tratamento fisioterapêutico convencional isolado

Protocolo de fortalecimento durante três meses
Investigar 0 efeito dos exercícios de estabilização postural na dor, força muscular e função

\section{Exercícios} terapêuticos de joelho estabilização postural $(\mathrm{n}=$ 22) $x$ exercícios terapêuticos de joelho $(n=20)$
Houve melhora do quadro clínico das voluntárias, redução da dor e da limitação funcional.

A adição de exercícios de estabilização postural a um programa de exercício de joelho de 6 semanas foi mais eficaz em melhorar a força, função e reduzir a dor

\section{0}




\section{DISCUSSÃO}

A SDFP causa desconforto ao paciente e, consequentemente, redução da sua funcionalidade. O tratamento conservador visa diminuir a queixa álgica apresentada e proporcionar melhor desempenho nas atividades de vida diária do indivíduo.

Embora os pacientes com SDFP apresentem dificuldades no controle dos movimentos dos MMII e tronco, em conjunto com a fraqueza muscular do quadril e do tronco, há dados limitados sobre os resultados biomecânicos de protocolos de reabilitação com enfoque na melhoria dessas deficiências (FERBER et al., 2015).

A este respeito, dentre as investigações existentes encontram-se estudos com comparações semelhantes (BALDON et al., 2014; FERBER et al., 2015; SAAD et al., 2018). Em pesquisa realizada em 2015 houve confrontamento dos resultados de um protocolo de fortalecimento com enfoque em quadril com um focado em joelho baseado em exercícios em CCA, CCF e exercícios de equilíbrio. No protocolo de quadril foram realizadas 3 séries dos exercícios variando de 10 a 15 repetições. No protocolo de joelho foram realizadas 3 séries variando de 10 a 30 repetições. Os dois protocolos foram realizados sem carga e os exercícios de agachamento utilizaram $90^{\circ}$ de flexão de quadril e joelhos. As intervenções foram realizadas durante 6 semanas com 3 atendimentos semanais. O protocolo do quadril foi mais eficaz. No entanto, o estudo não pôde afirmar que o tratamento baseado somente no fortalecimento é melhor que alguma outra intervenção, visto que não foi incluso um grupo controle (FERBER et al., 2015).

A intervenção baseada em exercícios de fortalecimento muscular do quadril e controle de movimento de membros inferiores e tronco é mais benéfica quando comparada a um programa de exercícios de fortalecimento do quadríceps isolado. Essa comprovação é resultado de pesquisa após um tratamento de 8 semanas com frequência de 3 vezes semanais, com intervalo mínimo de 24 horas entre as sessões onde houve a comparação entre um treinamento padrão (TP) com protocolo de quadril acrescentado de exercícios de controle de tronco (TEF) em mulheres atletas com faixa etária entre 18 e 30 anos. Ao longo do tratamento TP foram realizadas de 2 a 3 séries, de 12 a 20 repetições. A carga inicial foi de 50\% de 1RM, progredindo para 75\% 1RM a partir da 3ำ semana. Os exercícios na cadeira extensora - de 90 a 45--, leg-press - 0 a $45^{\circ}-$ e agachamento na parede - 0 a $60^{\circ}$ - foram aplicados em sessões que duraram de 75 a 90 minutos. No tratamento TEF, as séries variaram de 2 a 3, e 12 a 20 repetições. Na primeira semana de 
intervenção a carga foi de $20 \%$ de $1 \mathrm{RM}$ progredindo para $75 \%$ a partir da 3 semana. $\mathrm{O}$ exercício na cadeira extensora foi realizado de $90^{\circ}$ a $45^{\circ}$ com a sessão durrando de 90 a 120 minutos. Após 3 meses do tratamento os grupos foram reavaliados e o grupo TEF apresentou menos dor e maior melhora global e função física (BALDON et al., 2014).

O protocolo de quadril foi mais eficaz na redução de dor e aumento de força muscular (BALDON et al., 2014; FERBER et al., 2015) com diminuição de queixa álgica a partir da $3^{\text {a }}$ semana de intervenção, enquanto o protocolo joelho apresentou redução significativa a partir da 4⿳亠丷a semana. Ambas intervenções apresentaram aumento de força muscular ao final do tratamento.

Os exercícios de fortalecimento do quadril e do quadríceps melhoraram a cinemática dos membros inferiores no fim de 8 semanas de intervenção em pesquisa realizada com quarenta mulheres. As mesmas foram alocadas aleatoriamente em quatro grupos: exercícios de quadril, exercícios de quadríceps, alongamentos e controle (sem intervenção). Cada sessão teve duração de 50 minutos sendo realizada 2 vezes por semana, durante 8 semanas. Nessa comparação chegou-se à conclusão que as formas de tratamento mais comuns para pacientes com SDFP foram igualmente eficazes, não havendo diferenças significativas entre elas. Inclusive esse foi o único estudo que analisou de forma isolada o papel do alongamento muscular, demonstrando ser igualmente eficaz para alívio de dor quando comparado com a técnica de fortalecimento (SAAD et al., 2018).

Entretanto, em estudos comparativos de protocolos de joelho ao de quadril é necessário lembrar que alguns músculos são biarticulares, atravessando as articulações do joelho e do quadril, por isso o isolamento total de cada musculatura não parece possível (BALDON et al., 2014; FERBER et al., 2015; SAAD et al., 2018).

Em investigação realizada na Turquia (Yelvar et al., 2015), houve comparação de um protocolo de exercícios terapêuticos de joelho e estabilização postural (grupo 1) com um protocolo com apenas exercícios terapêuticos de joelho (grupo 2). Os exercícios de estabilização foram realizados 3 dias por semana, durante 6 semanas, sob supervisão de um fisioterapeuta. Os princípios de estabilização postural como a ativação do core, manutenção da coluna ereta e padrão respiratório diafragmático foram explicados antes do tratamento e os pacientes foram solicitados a cumprir estes durante o exercício. No grupo 2 foram prescritos exercícios terapêuticos do joelho à serem realizados em casa e 
pediu-se para que os participantes escrevessem um diário de exercícios concluídos. Os pacientes foram contatados 4 vezes por semana einstruídos a realizar o programa de exercícios em casa 3 vezes ao dia com 10 repetições para cada exercício usando seu próprio peso corporal durante as primeiras 2 semanas e, posteriormente, utilizando uma faixa de resistência elástica para aumentar a força.

Os indivíduos foram reavaliados pós intervenção e após 12 semanas. Os dois grupos apresentaram resultados positivos nos quesitos dor, flexibilidade, função e força, mas com diferenças significativas a favor do grupo 1. Sendo assim, sugere-se acrescentar exercícios de estabilização postural a um programa de exercício de joelho, pois o mesmo foi mais eficaz em melhorar a força, função e redução da queixa dolorosa. É importante destacar que, o fato de o grupo 2 ter realizado os exercícios em casa sem a supervisão de um profissional deixa dúvidas quanto à eficácia na realização do movimento e ao número de repetições propostas, visto que o paciente pode acabar fazendo compensações ou não realizando a quantidade proposta. No entanto, a presença de resultados positivos reafirma a importância da orientação do paciente que influenciará no sucesso da reabilitação e na prevenção da recidiva da patologia.

$\mathrm{Na}$ comparação entre um protocolo de fisioterapia e Kinesio-Taping® (grupo experimental) à um grupo que recebeu apenas fisioterapia (grupo controle) (Sanchez et al., 2017), a intervenção foi realizada durante 30 dias com frequência de 2 vezes por semana, com a sessão de 50 minutos. Os dois grupos realizaram fortalecimento de quadríceps e abdutores de quadril, alongamentos de reto femoral, adutores, isquiotibiais, tríceps sural e tensor da fáscia lata. A estimulação elétrica funcional foi utilizada em ambos os grupos no VMO para corrigir posicionamento patelar. Porém, o estudo não relata quantas vezes e durante quando tempo foi utilizado. Em relação à funcionalidade e equilíbrio, não houve diferença significativa entre os grupos, quanto a dor foi verificado que o grupo experimental teve uma melhora significativa $(p=0,012)$ na Escala Visual Analógica (EVA) proporcionando maior alívio aos pacientes, demostrando que a Kinesio Taping® pode ser complementar a cinesioterapia. Essa técnica tornou-se popular apenas a alguns anos havendo um número limitado de ensaios clínicos com resultados ainda controversos, justificando provavelmente à carência de estudos voltados ao seu uso na SDFP e, consequentemente, limitando a comprovação da eficácia clínica deste recurso. 
Em um acompanhamento durante 12 semanas, 3 vezes por semana, aplicou-se exercícios de fortalecimento em MMII após padronizar com estresse articular indivíduos acometidos pela síndrome. Ao fim da intervenção foi comparado o grupo acometido pela síndrome ao grupo de indivíduos saudáveis e que não receberam o tratamento. As séries de repetições com cargas progressivas foram baseadas no American College of Sports Medicine de progressão do treinamento de resistência, sendo proporcionais ao peso corporal. Na primeira semana os exercícios foram realizados sem carga, na segunda foi adicionado a carga de $5 \%$ do peso corporal, adicionando $5 \%$ a cada duas semanas. 0 fortalecimento muscular em CCF foi realizado em $90^{\circ}$ de flexão de quadril e joelhos. Após três meses de acompanhamento os indivíduos do grupo SDFP foram comparados com o grupo controle, sugerindo que o tratamento deva incluir exercícios de fortalecimento em CCF para membros inferiores, de forma individualizada, com aumento progressivo da carga e orientações adequadas de posicionamento para realização dos exercícios (Souza et al., 2017).

Um dos sintomas no paciente com SDFP é a dor no joelho ao passar muito tempo sentado, podendo ocasionar um incômodo ao retornar à posição ortostática e realizar a marcha. Em um estudo que avaliou o uso de órtese patelofemoral durante 6 semanas houve uma redução de 59,6\% na dor e aumento na velocidade da marcha e comprimento do passo demonstrando um efeito positivo do uso de órteses, fornecendo evidências para apoiar o seu uso como tratamento conservador (ARAZPOUR et al., 2013).

O estudo de Glaviano e Saliba recrutou 22 pacientes separados em dois grupos para receberem tratamentos diferentes. Em um dos grupos os participantes receberam estimulação nervosa elétrica percutânea no padrão bifásico de onda quadrada assimétrica sobre o VMO, glúteo médio (GM), grupo muscular de adutores e isquiotibiais durante 15 minutos. Para o tratamento simulado, nos outros sujeitos da pesquisa, foi aplicado o sistema de eletroterapia com configurações idênticas, no entanto, o grupo recebeu um tratamento de amplitude de $1 \mathrm{~mA}$, que é o estímulo mínimo disponível com a unidade. $\mathrm{O}$ tratamento simulado também durou 15 minutos, e os participantes foram informados que eles estavam recebendo um tratamento subsensorial. No final da intervenção, 5 tarefas adicionais de agachamento unipodal e step-down lateral foram realizadas, para coletar dados para eletromiografia e EVA, e comparar com os dados colhidos antes da intervenção, comprovando que um único tratamento da estimulação nervosa elétrica 
percutânea produziu uma diminuição imediata da dor e aumentou a porcentagem de ativação GM durante tarefas funcionais. A diminuição imediata da dor favorece a reabilitação e a execução das tarefas funcionais, reduzindo algum receio que o paciente possa apresentar durante atividades que costumam ser dolorosas. Ao diminuir a dor pode-se progredir no treinamento funcional ou aumentar os conjuntos e repetições durante o processo de reabilitação (GLAVIANO \& SALIBA, 2016).

$\mathrm{Na}$ pesquisa de Østerås e Torstensen, para os pacientes de grupos experimental e controle, a carga e a amplitude de movimento foram graduadas para tornar o programa adequado e realizável com o mínimo de desconforto possível. A intervenção foi realizada 3 vezes por semana durante 12 semanas. Um ano após a conclusão, as diferenças dos grupos foram mantidas e até aumentaram, ou seja, o GE continuou a melhorar, enquanto - GC, recaiu com um aumento de dor e disfunção. Uma abordagem extensa de fisioterapia pode parecer dispendiosa, porém por ser eficiente, haverá uma redução de ausências de trabalho e, quanto mais o paciente se mantiver saudável, o mesmo se tornará menos dependente de tratamentos e dos serviços de saúde, diminuindo gastos tanto individuais quanto públicos. A reabilitação com alta dose e alta repetição se mostrou altamente eficaz, visto que demonstrou efeitos a longo prazo com em pacientes no que diz respeito à dor e aos desfechos funcionais. Os parâmetros de dosagem no GC foram classificados de acordo com os princípios tradicionais de treinamento de força, comumente prescritos para pacientes com SDFP. Porém, além de ter um número de participantes relativamente baixo, 28 participantes, o estudo não relata exercícios, ângulos e número de repetições cada grupo realizou, dessa forma não favorece que seja aplicado de forma fidedigna na prática clínica, devido ausência de parâmetros que poderiam ser reproduzidos durante a intervenção fisioterapêutica (ØSTERÅS et al., 2013).

Alguns autores sugerem que a associação de técnicas é mais benéfica para pacientes com SDFP (SANCHEZ et al., 2017; YELVAR et al., 2015). No entanto, a reabilitação mais indicada e efetiva deve ser baseada nos déficits observados na avaliação de cada indivíduo. A duração das intervenções nos estudos encontrados variou entre 4 a 12 semanas, com frequência média de 3 vezes na semana sugerindo que o tempo médio de intervenção seja este (ØSTERÅS et al., 2013; SOUZA et al., 2017; ARAZPOUR et al., 2013; Baldon et al., 2014; GLAVIANO, 2016; SANCHEZ et al., 2017; YELVAR et al., 2015). 


\section{CONSIDERAÇÕES FINAIS}

A partir da elaboração desse estudo, é possível demonstrar que as técnicas que a fisioterapia dispõe aliviam dor, fortalecem e melhoram flexibilidade dos principais e músculos acometidos pela SDFP. Resultados positivos foram atribuídos a exercícios de alongamentos, agachamentos e exercícios isométricos de quadríceps. Quatro dos programas de exercícios incluíram exercícios para quadril e dois estudos sugerem adicionar exercícios para estabilização de tronco. O tempo médio de intervenção variou de 4 a 12 semanas com frequência de três vezes semanais. Mesmo quando comparada a outra intervenção, cada técnica apresentou seus benefícios, porém a modalidade em destaque foi a cinesioterapia com enfoque em fortalecimento das musculaturas de quadril e joelho.

\section{REFERENCIAS}

ARAZPOUR, Mokhtar et al. The effect of patellofemoral bracing on walking in individuals with patellofemoral pain syndrome. Prosthetics and Orthotics International. Islamic Republic of Iran, v. 37, n. 6, fev. 2013, p. $465-470$.

ASSIS, Lívia. A intervenção fisioterapêutica na síndrome da dor femoropatelar nas corridas de rua: uma revisão bibliográfica. Revista Especialize On-line. Rio de Janeiro, vol. 01, n. 10, dez. 2015, p. 1-10.

BALDON, Rodrigo, et al. Effects of functional stabilization training on pain, function, and lower extremity biomechanics in women with patellofemoral pain: a randomized clinical trial. J Orthop Sports Phys Ther, v. 44, n. 4, abr. 2014, p. 240-248.

BESSA Sintia et al. A eficácia da bandagem funcional na síndrome da dor femoropatelar. Revista Faculdade Montes Belo, vol. 9, n. 1, 2016, p. 20-173.

CAMPOS, lara, et al. Tratamento fisioterapêutico na síndrome da dor patelofemoral: uma revisão da literatura. Revista Movimenta ISSN: 1984-4298.Goiás, vol. 6, n. 3, 2013.

FERBER, Reed et al. Strengthening of the Hip and Core Versus Knee Muscles for the Treatment of Patellofemoral Pain: A Multicenter Randomized Controlled Trial. Journal of Athletic Training. Canadá, vol. 50, n. 4, abr. 2015 , p. 366-377.

GLAVIANO Neal, SALIBA, Susan. Immediate Effect of Patterned Electrical Neuromuscular Stimulation on Pain and Muscle Activation in Individuals With Patellofemoral Pain. Journal of Athletic Training. Charlottesville, vol. 51, n. 2, fev. 2016, p. 118-128. 
PEREIRA JÚNIOR, Altair; LIMA, Walter. Avaliação da síndrome da dor patelofemoral em mulheres. Revista Brasileira de Pesquisa em Saúde. Fortaleza, vol 24, n. 1, jan/mar. 2011, p. 5-9.

NETO, Luíz; CAVALCANTE, Idília; JÚNIOR, Manoel. Abordagens fisioterapêuticas na síndrome da dor patelofemoral: revisão de literatura. ConScientia e Saúde. Teresina, vol. 13, n. 3, ago. 2014, p. 471-479.

OLIVEIRA, Letícia Villani de et al. Muscle strength analysis of hip and knee stabilizers in individuals with Patellofemoral Pain Syndrome. Fisioter. Pesqui. São Paulo, v. 21, n. 4, dez. 2014, p. 327-332.

ØSTERÅS, Berit et al. Dose-response effects of medical exercise therapy in patients with patellofemoral pain syndrome: a randomised controlled clinical trial. Physiotherapy. Trondheim, vol. 99, n. 4, 2013, p. 311-316.

PIAZZA, Lisiane et al. Sintomas e limitações funcionais de pacientes com síndrome da dor patelofemoral. Rev. dor, São Paulo, v. 13, n. 1, mar. 2012, p. 50-54.

ROQUE, Vanessa et al. Síndrome Femoro-Patelar. Revista da Sociedade Portuguesa de Medicina Física e de Reabilitação. Portugal, vol. 22, n. 2, nov. 2012, p. 53-61.

SAAD, Marcelo et al. Is hip strengthening the best treatment option for females with patellofemoral pain? A randomized controlled trial of three different types of exercises. Brazilian Journal of Physical Therapy, v. 22, n. 5, mar. 2018, p. 408-416.

SANCHEZ, Hugo et al. Influence of the treatment of the Kinesio-taping® technique on pain and functionality in patients with Patellofemoral Pain Syndrome. O Mundo da Saúde. São Paulo, v.41, n.1, mar. 2017, p. 48-56.

SANTOS, Alan; OLIVEIRA, Alexsandro. Avaliação biomecânica dos músculos do quadril em indivíduos com síndrome femoropatelar: revisão da literatura. Corpus scientia. Rio de Janeiro, v. 11, n. 2, dez. 2015, p. 1-8.

SANTOS, Luciano et al. A utilização da reabilitação e exercícios terapêuticos na síndrome da dor femoropatelar- uma revisão sistemática. Revista Brasileira de Prescrição e Fisiologia do Exercício. São Paulo, vol. 07, n. 39, mai/jun. 2013, p. 225-236.

SIZÍNIO, Herbert. Ortopedia e Traumatologia: princípios e prática. 4. ed. Porto Alegre: Artmed; 2009.

SOUZA, Gabriela et al. Eficácia de um protocolo de exercícios em cadeia cinética fechada para indivíduos com dor femoropatelar. ConScientiae Saúde. Araranguá, vol. 16, n. 4, nov. 2017, p. 393-401.

YELVAR, Gül et al. The effect of postural stabilization exercises on pain and function in females with patellofemoral pain syndrome. Acta orthopaedica et traumatologica turcica. Ankara, v. 49, n. 2, 2015, p. 166-74. 
ZAMBOTI, Camila et al. Análise da dor, capacidade funcional, força muscular e equilíbrio em mulheres jovens com Síndrome da Dor Patelofemoral. Fisioter. mov., Curitiba, v. 30, n. 3, Set. 2017, p. 433-441.

ZANARDI, Cláudia; LIMA, Malu. Intervenção fisioterapêutica em pacientes portadores da síndrome femoropatelar. Revista Saúde Meio Ambiente, v. 1, n. 1, jun. 2012, p. 163-172. 